\title{
Late-Time Magnetogenesis Driven by Axionlike Particle Dark Matter and a Dark Photon
}

\author{
Kiwoon Choi, ${ }^{1, *}$ Hyungin Kim, ${ }^{2,1, \dagger}$ and Toyokazu Sekiguchi ${ }^{3,1, \$}$ \\ ${ }^{1}$ Institute for Basic Science, Center for Theoretical Physics of the Universe, Daejeon 34051, South Korea \\ ${ }^{2}$ Department of Particle Physics and Astrophysics, Weizmann Institute of Science, Rehovot 7610001, Israel \\ ${ }^{3}$ Research Center for the Early Universe (RESCEU), Graduate School of Science, The University of Tokyo, \\ Tokyo 113-0033, Japan
}

(Received 12 March 2018; revised manuscript received 18 May 2018; published 17 July 2018)

We propose a mechanism generating primordial magnetic fields after the $e^{+} e^{-}$annihilations. Our mechanism involves an ultralight axionlike particle (ALP) which constitutes the dark matter and a dark $U(1)_{X}$ gauge boson introduced to bypass the obstacle placed by the conductivity of cosmic plasma. In our scheme, a coherently oscillating ALP amplifies the dark photon field, and part of the amplified dark photon field is concurrently converted to the ordinary magnetic field through the ALP-induced magnetic mixing. For the relevant ALP mass range $10^{-21} \mathrm{eV} \lesssim m_{\phi} \lesssim 10^{-17} \mathrm{eV}$, our mechanism can generate $B \sim 10^{-24} \mathrm{G}\left(m_{\phi} / 10^{-17} \mathrm{eV}\right)^{5 / 4}$ with a coherent length $\lambda \sim\left(m_{\phi} / 10^{-17} \mathrm{eV}\right)^{-1 / 2} \mathrm{kpc}$, which is large enough to provide a seed of the galactic magnetic fields. The mechanism also predicts a dark $U(1)_{X}$ electromagnetic field $E_{X} \sim B_{X} \sim 80 \mathrm{nG}\left(m_{\phi} / 10^{-17} \mathrm{eV}\right)^{-1 / 4}$, which can result in interesting astrophysical or cosmological phenomena by inducing the mixings between the ALP, ordinary photon, and dark photon states.

DOI: 10.1103/PhysRevLett.121.031102

The origin of the primordial magnetic fields is one of the long-standing problems in modern cosmology. In a variety of cosmological scales, magnetic fields are observed [1-3]. For instance, radio observations have revealed that magnetic fields of $\mathcal{O}(1-10) \mu \mathrm{G}$ are inherent in the galaxies and clusters of galaxies, which might originate from a primordial seed field [4]

$$
B_{\text {seed }} \gtrsim \mathcal{O}\left(10^{-30}\right) \mathrm{G} \quad \text { with } \quad \lambda \gtrsim 0.1 \mathrm{kpc},
$$

which is amplified later by the dynamo mechanism [5], where $\lambda$ denotes the coherent length of the corresponding $B$ fields. More recently, the existence of magnetic fields in cosmic voids has been inferred from the observations of $\mathrm{TeV}$ blazars [6-12]. Those observations have found a lack of secondary gamma rays in the $\mathrm{GeV}$ range, which ought to be emitted by the electron-positron pairs produced from the collision of the primary gamma rays with extragalactic background light. This can be explained if there exist magnetic fields at intergalactic voids

$$
B_{\text {void }} \times \min [1, \sqrt{\lambda / 0.1 \mathrm{Mpc}}] \gtrsim \mathcal{O}\left(10^{-19}-10^{-16}\right) \mathrm{G},
$$

Published by the American Physical Society under the terms of the Creative Commons Attribution 4.0 International license. Further distribution of this work must maintain attribution to the author(s) and the published article's title, journal citation, and DOI. Funded by SCOAP ${ }^{3}$. which deflect the trajectory of the produced electronpositron pairs away from the direction of the primary gamma rays [3].

So far, a number of models are proposed for cosmological magnetogenesis in the early Universe (for a recent review, see, e.g., Ref. [3]). An interesting and extensively studied possibility is the inflationary magnetogenesis scenario [13-19]. In this scenario, magnetic fields created inside the horizon can be stretched out to superhorizon scales and can have a comoving correlation length comparable to the current cosmological scales. However, inflationary magnetogenesis often suffers from the backreaction by the produced magnetic fields, which spoils the dynamics of inflaton or generates too large non-Gaussianity in primordial perturbations [15-17] [these problems can be circumvented if magnetogenesis takes place after the cosmic microwave background (CMB) scales exit the horizon [18,19]]. There are other magnetogenesis scenarios, for instance, based on phase transition in the early Universe [20,21]; however, those scenarios are still lacking concrete realization (see, also, Ref. [22] for a generation of primordial magnetic field with QCD axion prior to the QCD phase transition).

Another interesting but less explored possibility is a latetime magnetogenesis which takes place well after big bang nucleosynthesis (BBN). Such a late-time scenario would have better prospects to give a large coherence length of the produced $B$ fields and also may come up with concrete predictions, as the early Universe after the BBN is highly constrained. In this Letter, we propose a novel mechanism 
of late-time magnetogenesis, which occurs after the $e^{+} e^{-}$ annihilations.

Our mechanism involves an ultralight axionlike particle (ALP) $\phi$ and a dark $U(1)_{X}$ gauge field $X_{\mu}$, whose dynamics is described by the Lagrangian

$$
\begin{aligned}
\mathcal{L}= & \frac{1}{2} \partial_{\mu} \phi \partial^{\mu} \phi-\frac{1}{2} m_{\phi}^{2} \phi^{2}-\frac{1}{4} F_{\mu \nu} F^{\mu \nu}-\frac{1}{4} X_{\mu \nu} X^{\mu \nu} \\
& -\frac{g_{A A}}{4 f} \phi F_{\mu \nu} \tilde{F}^{\mu \nu}-\frac{g_{X X}}{4 f} \phi X_{\mu \nu} \tilde{X}^{\mu \nu}-\frac{g_{A X}}{2 f} \phi F_{\mu \nu} \tilde{X}^{\mu \nu} \\
& +J^{\mu} A_{\mu},
\end{aligned}
$$

where $F_{\mu \nu}=\partial_{\mu} A_{\nu}-\partial_{\nu} A_{\mu}$ and $X_{\mu \nu}=\partial_{\mu} X_{\nu}-\partial_{\nu} X_{\mu}$ are the $U(1)_{\mathrm{em}}$ and $U(1)_{X}$ field strengths, $\tilde{F}_{\mu \nu}$ and $\tilde{X}_{\mu \nu}$ are their duals, $J^{\mu}$ is the standard electromagnetic current, and $f$ is a dimensionful parameter describing the initial ALP misalignment:

$$
f \equiv \phi_{\text {initial }}
$$

In the expanding Universe with the metric

$$
d s^{2}=a^{2}(\tau)\left(d \tau^{2}-d x^{2}\right),
$$

the equations of motion for the ALP and $U(1)$ gauge bosons are given by

$$
\begin{aligned}
\ddot{\boldsymbol{\phi}}+ & 2 \mathcal{H} \dot{\phi}-\nabla^{2} \phi+a^{2} m_{\phi}^{2} \phi=-\frac{1}{a^{2}}\left(\frac{g_{A A}}{f} \dot{\boldsymbol{A}} \cdot \nabla \times \boldsymbol{A}\right. \\
& \left.+\frac{g_{X X}}{f} \dot{\boldsymbol{X}} \cdot \nabla \times \boldsymbol{X}+\frac{g_{A X}}{f}(\dot{\boldsymbol{A}} \cdot \nabla \times \boldsymbol{X}+\dot{\boldsymbol{X}} \cdot \nabla \times \boldsymbol{A})\right), \\
\ddot{\boldsymbol{A}} & +\sigma(\dot{\boldsymbol{A}}+\boldsymbol{v} \times(\nabla \times \boldsymbol{A}))+\nabla \times(\nabla \times \boldsymbol{A}) \\
& =\frac{g_{A A}}{f}(\dot{\phi} \nabla \times \boldsymbol{A}-\nabla \phi \times \dot{\boldsymbol{A}})+\frac{g_{A X}}{f}(\dot{\phi} \nabla \times \boldsymbol{X}-\nabla \phi \times \dot{\boldsymbol{X}}), \\
\ddot{\boldsymbol{X}} & +\nabla \times(\nabla \times \boldsymbol{X})=\frac{g_{X X}}{f}(\dot{\phi} \nabla \times \boldsymbol{X}-\nabla \phi \times \dot{\boldsymbol{X}}) \\
& +\frac{g_{A X}}{f}(\dot{\phi} \nabla \times \boldsymbol{A}-\nabla \phi \times \dot{\boldsymbol{A}}),
\end{aligned}
$$

where we used the temporal gauge $A_{\mu}=(0, \boldsymbol{A}), X_{\mu}=(0, \boldsymbol{X})$. Here, the dots denote the derivatives with respect to the conformal time $\tau, \mathcal{H}=\dot{a} / a=a H$ is the conformal Hubble expansion rate, and finally, Ohm's law $\boldsymbol{J}=\sigma(\boldsymbol{E}+\boldsymbol{v} \times \boldsymbol{B})$ is used for the equation of $\boldsymbol{A}$, where $\sigma$ is the conformal conductivity of the cosmic plasma, and $v$ is a fluid velocity field.

The following is a brief summary of how our mechanism works and what the underlying assumptions are. At $\tau_{\text {osc }}$ when $3 H\left(\tau_{\text {osc }}\right) \simeq m_{\phi}$, the ALP $\phi$ commences to oscillate to form the dark matter. Coherently oscillating $\phi$ causes a tachyonic instability of $X_{\mu}$ through the coupling $g_{X X}$ and amplifies the dark photon field strength as

$$
E_{X} \simeq B_{X} \simeq 83 \mathrm{nG}\left(m_{\phi} / 10^{-17} \mathrm{eV}\right)^{-1 / 4}
$$

for $g_{X X} \gtrsim \mathcal{O}(10)$. For an efficient amplification, we assume that $X_{\mu}$ is strictly massless, and there is no light $U(1)_{X}$-charged particle. In the presence of the magnetic mixing coupling $g_{A X}$ [23], the amplified $E_{X} \sim B_{X}$ are partly converted into the ordinary magnetic fields generating

$$
B \simeq 1.7 \times 10^{-24} \mathrm{G}\left(m_{\phi} / 10^{-17} \mathrm{eV}\right)\left(\frac{g_{A X} / f}{10^{16} \mathrm{GeV}}\right),
$$

with a coherent length $\lambda \sim\left(m_{\phi} / 10^{-17} \mathrm{eV}\right)^{-1 / 2} \mathrm{kpc}$. The conversion of $X_{\mu}$ to $A_{\mu}$ is most efficient when the conductivity $\sigma \propto n_{e}$ is minimized, which happens when the electrons and positions are annihilated enough, so the electron density is suppressed as $n_{e} \sim n_{\text {baryon }} \sim 10^{-9} n_{\gamma}$. This happens when $T \lesssim 20 \mathrm{keV}$. For this reason, we consider the ALP mass range

$$
10^{-21} \mathrm{eV} \lesssim m_{\phi} \lesssim 10^{-17} \mathrm{eV}
$$

for which ALP begins to oscillate at $T_{\text {osc }} \simeq 100 \mathrm{keV} \times$ $\left(m_{\phi} / 10^{-17} \mathrm{eV}\right)^{1 / 2}$, so that magnetogenesis occurs at $T \sim T_{\text {osc }} / 5 \lesssim 20 \mathrm{keV}$ as desired. Here, the lower bound on $m_{\phi}$ is imposed to be compatible with the Lyman- $\alpha$ constraint on ultralight ALP dark matter [24]. Then, the temperature range of our mechanism is

$$
200 \mathrm{eV} \lesssim T \lesssim 20 \mathrm{keV},
$$

for which the conductivity is determined by the Thomson scattering as [25-27]

$$
\sigma_{\mathrm{phy}}=\frac{\sigma}{a} \simeq \frac{135 \zeta(3)}{e^{2} \pi^{3}} \frac{m_{e}^{2}}{T} \frac{n_{e}}{n_{\gamma}} .
$$

Our model (3) generically involves three ALP couplings: $g_{A A}, g_{A X}$, and $g_{X X}$. To check the feasibility of our mechanism, we list current observational bounds on the ALP couplings [28]. For an ultralight ALP, major constraints on $g_{A A}$ come from astrophysical observations based on the photon-ALP conversion [30], e.g., x-ray observations [31-35], quasar spectra [36], cosmological tests of the distance-duality relation [37,38], and CMB spectral distortions [39-42]. In our case, the strongest bound on $g_{A A}$ comes from x-ray observation [33] yielding $g_{A A} / f \lesssim 1.5 \times 10^{-12} \mathrm{GeV}^{-1}$. Constraints on $g_{A X}$ can be drawn also from the ALP-photon mixing induced by background $B_{X}$. We find the strongest bound on $g_{A X}$ in our case comes from the $\mathrm{CMB}$ spectral distortion yielding $[40,43]$

$$
\frac{g_{A X}}{f} \lesssim 10^{-16} \mathrm{GeV}^{-1}\left(\frac{B_{X}}{100 \mathrm{nG}}\right)^{-1}
$$


The coupling $g_{X X} / f$ is far less constrained, as it involves only the dark photon fields and can have a value large enough to implement our mechanism.

To motivate the introduction of $X_{\mu}$, let us briefly discuss the generation of magnetic field in the absence of $X_{\mu}$. When $\sigma_{\text {phy }} \gg m_{\phi}$, we can approximate the equation of motion for the photon field as $\sigma \dot{A}_{k} \approx g_{A A} k \dot{\phi} A_{k} / f$ in the Fourier space, where $k$ is the comoving wave number. This allows a solution for the magnetic field as $A_{k}=A_{\mathrm{k}, \mathrm{vac}} \exp \left[\int d \tau^{\prime} \Gamma\right]$ where $A_{\mathrm{k}, \mathrm{vac}}$ is the vacuum fluctuation of the gauge field, and $\Gamma=g_{A A} k \dot{\phi} /(\sigma f)$ is the magnetic field production rate. Then, the magnetic field production for the unit Hubble time at $\tau \sim \tau_{\text {osc }}$ is estimated as

$$
\frac{1}{\mathcal{H}} \frac{\dot{\boldsymbol{A}}}{\boldsymbol{A}}=\frac{\Gamma}{\mathcal{H}} \lesssim\left(\frac{g_{A A}}{f}\right)^{2} \frac{m_{\phi} f^{2}}{\sigma_{\text {phy }}} \lesssim 5 \times 10^{-9},
$$

where $k$ is within the instability regime, i.e., $k / a \lesssim$ $\mathcal{O}\left(g_{A A} m_{\phi}\right)$. Here, we used $\dot{\phi} / \mathcal{H} f=\mathcal{O}(1)$ at $\tau \sim \tau_{\text {osc }}$ and $\sigma_{\text {phy }}$ given by Eq. (11), together with the bounds on $g_{A A} / f$ and $m_{\phi}$, while assuming $f \lesssim M_{\mathrm{Pl}}$ which is necessary to avoid a too large relic mass density of $\phi$. The above production rate is too weak to yield any appreciable amount of $B$ fields, which is essentially due to the huge suppression by $m_{\phi} / \sigma_{\text {phy }} \lesssim 2 \times 10^{-17}$.

With the dark photon field $X_{\mu}$, we can make magnetogenesis much more efficient. As the cosmic plasma is neutral to $U(1)_{X}, X_{\mu}$ can be freely amplified by the tachyonic instability caused by the oscillating $\phi$, and this exponential amplification can compensate for much of the suppression by $m_{\phi} / \sigma_{\text {phys. }}$. In the following, we describe our magnetogenesis mechanism and present some of the key results, while leaving more detailed study to the forthcoming work [45].

Right after $\tau_{\text {osc }}$, when the energy density $\rho_{X}$ of $X_{\mu}$ is negligible compared to $\rho_{\phi}$, $\phi$ evolves as

$$
\theta \equiv \frac{\phi}{f} \approx\left(\frac{a(\tau)}{a\left(\tau_{\mathrm{osc}}\right)}\right)^{-3 / 2} \cos \left[m_{\phi}\left(t-t_{\mathrm{osc}}\right)\right],
$$

where $t=a(\tau) \tau / 2$. In this stage, $\phi$ is approximately homogeneous, and the backreaction from $A_{\mu}$ can be ignored. Then, the equation of motion of $X_{\mu}$ in the momentum space is approximated by

$$
\ddot{\boldsymbol{X}}_{k \pm}+k\left(k \mp g_{X X} \dot{\theta}\right) \boldsymbol{X}_{k \pm} \simeq 0,
$$

where the subscript \pm denotes the helicity. This shows that under the oscillating $\phi$, one of the helicity states of $\boldsymbol{X}_{k}$ experiences a tachyonic instability for certain range of $k$, and the vacuum fluctuations of $\boldsymbol{X}_{k}$ in this range of $k$ are exponentially amplified to be a stochastic classical field.

At a certain time $\tau_{X}>\tau_{\text {osc }}, \rho_{X}$ becomes comparable to $\rho_{\phi}$, where $\tau_{X} / \tau_{\text {osc }}=a\left(\tau_{X}\right) / a\left(\tau_{\text {osc }}\right)$ is determined mostly by the coupling $g_{X X}$. Around this time, the initial energy density of the zero momentum mode of $\phi$ is converted mostly to $\rho_{X}$ and also partly to the energy density of nonzero momentum modes of $\phi$. As was shown in Ref. [46], the dark photon field production is particularly efficient for $g_{X X} \gtrsim \mathcal{O}(10)$, and in this Letter, we will use $g_{X X}=100$ as a benchmark point for explicit analysis. The ordinary electromagnetic field $A_{\mu}$ is produced also around this time by the magnetic mixing coupling $g_{A X}$. As the conductivity dominates over other factors, the production is described by the following approximate equation of motion

$$
\sigma \dot{\boldsymbol{A}} \simeq g_{A X}(\dot{\theta} \nabla \times \boldsymbol{X}-\nabla \theta \times \dot{\boldsymbol{X}}),
$$

where the effects of nonzero momentum modes of $\phi$ are included.

For a more quantitative analysis, we define

$$
\begin{aligned}
& r(\tau) \equiv \frac{\left\langle\rho_{\phi}\right\rangle(\tau)}{\left(\rho_{\phi}\right)_{g=0}(\tau)}, \\
& \epsilon(\tau) \equiv \frac{a(\tau)}{a\left(\tau_{\mathrm{osc}}\right)} \frac{\left\langle\rho_{X}\right\rangle(\tau)}{\left(\rho_{\phi}\right)_{g=0}(\tau)}, \\
& b(\tau) \equiv \frac{1}{g_{A X}} \frac{\sqrt{\left\langle\rho_{A}\right\rangle(\tau)}}{\sqrt{\left\langle\rho_{X}\right\rangle(\tau)}},
\end{aligned}
$$

where $\langle\rho\rangle$ denotes the spatially averaged energy density, and $\left(\rho_{\phi}\right)_{g=0}$ is the homogeneous energy density of $\phi$ in the absence of gauge field production, i.e., when $g_{A A}=g_{X X}=$ $g_{A X}=0$. The backreaction from $A_{\mu}$ can be safely ignored for $g_{A A}$ and $g_{A X}$ satisfying the observational bounds. Then, the evolutions of $r$ and $\epsilon$ are determined mostly by $g_{X X}$, while being insensitive to other model parameters. On the other hand, as we will see below, the evolution of $b(\tau)$ depends significantly on $m_{\phi}$. Obviously, in the early stage at $\tau<\tau_{X}, r \simeq 1$, and $\epsilon$ is negligibly small. In the intermediate stage at $\tau \simeq \tau_{X}, r$ drops to a value which is an order of magnitude smaller than unity, while $\epsilon$ rises to a value of order unity. In the final stage at $\tau \gg \tau_{X}$, the three fields $\phi$, $X_{\mu}$, and $A_{\mu}$ are decoupled from each other and freely evolve. As a result, the energy densities evolve as $\left\langle\rho_{\phi}\right\rangle \propto$ $1 / a^{3}$ and $\left\langle\rho_{X, A}\right\rangle \propto 1 / a^{4}$, and $r(\tau), \epsilon(\tau)$, and $b(\tau)$ all approach some constants.

As $X_{\mu}$ is exponentially amplified by the coupling $g_{X X}$, it strongly backreacts to the evolution of $\phi$ and develops an inhomogeneous part of $\phi$ for $\tau \gtrsim \tau_{X}$. A lattice simulation is required for a quantitative analysis of the evolution of our system. Yet, the dependence of the final results on $m_{\phi}$ and $f$ can be determined by simple dimensional analysis. For this, let us first note that $r, \epsilon$, and also $a\left(\tau_{\text {osc }}\right) / a\left(\tau_{X}\right)$ are insensitive to $m_{\phi}$ and $f$ [47]. We then find the following simple power-law dependences of the relevant quantities on $m_{\phi}$ and $f$, 


$$
\begin{aligned}
a\left(\tau_{X}\right) & \propto \tau_{X} \propto 1 / T_{X} \propto a\left(\tau_{\mathrm{osc}}\right) \propto m_{\phi}^{-1 / 2} \\
B_{X}^{2} & \propto a^{4}\left\langle\rho_{X}\right\rangle\left(\tau_{X}\right) \propto a^{4}\left\langle\rho_{\phi}\right\rangle\left(\tau_{X}\right) \propto a^{4}\left(\tau_{X}\right) m_{\phi}^{2} f^{2} \propto f^{2} \\
k_{*} & \sim g_{X X} \dot{\theta}\left(\tau_{X}\right) \propto a\left(\tau_{X}\right) m_{\phi} \propto m_{\phi}^{1 / 2} \\
\sigma\left(\tau_{X}\right) & =a\left(\tau_{X}\right) \sigma_{\mathrm{phy}}\left(\tau_{X}\right) \propto a\left(\tau_{X}\right) / T\left(\tau_{X}\right) \propto m_{\phi}^{-1}
\end{aligned}
$$

where $B_{X}^{2}=\left\langle|\nabla \times \boldsymbol{X}|^{2}\right\rangle$, and $k_{*}$ denotes the characteristic wave number of the produced $X_{\mu}$ and $A_{\mu}$. One can also infer from Eq. (16) that

$$
b\left(\tau_{X}\right) \propto \tau_{X} \dot{\theta}\left(\tau_{X}\right) k_{*} / \sigma\left(\tau_{X}\right) \propto m_{\phi}^{3 / 2},
$$

where we used the parametric dependences listed in Eq. (18).

From Eq. (17), the relic mass density of $\phi$ is determined as

$$
\Omega_{\phi} h^{2}=r\left(\tau_{0}\right)\left(\Omega_{\phi} h^{2}\right)_{g=0} \simeq 0.5 r\left(\tau_{0}\right) m_{-17}^{1 / 2} f_{16}^{2},
$$

where $\left(\Omega_{\phi} h^{2}\right)_{g=0}$ is the relic density in the absence of gauge field production, $\tau_{0}$ is the conformal time at present, and $m_{-17} \equiv m_{\phi} / 10^{-17} \mathrm{eV}, f_{16} \equiv f / 10^{16} \mathrm{GeV}$. The produced dark photon field and its energy density can be parametrized as

$$
\begin{gathered}
B_{X}\left(\tau_{0}\right) \simeq 21 \mathrm{nG} \times \sqrt{\frac{\epsilon\left(\tau_{0}\right)}{r\left(\tau_{0}\right)} \frac{\Omega_{\phi} h^{2}}{m_{-17}^{1 / 2}}}, \\
\Delta N_{\mathrm{eff}} \simeq 3.6 \times 10^{-4} \frac{\epsilon\left(\tau_{0}\right)}{r\left(\tau_{0}\right)} \frac{\Omega_{\phi} h^{2}}{m_{-17}^{1 / 2}} .
\end{gathered}
$$

Taking the ALP mass dependence of $b$ in Eq. (19), we can parametrize also the present value of the produced $B$ fields as

$B\left(\tau_{0}\right)=\left(3 \times 10^{-8} \mathrm{G}\right) \bar{g}_{-16} m_{-17} \Omega_{\phi} h^{2} \frac{\sqrt{\epsilon\left(\tau_{0}\right)}}{r\left(\tau_{0}\right)}\left(\frac{b\left(\tau_{0}\right)}{m_{-17}^{3 / 2}}\right)$,

where $\bar{g}_{-16} \equiv g_{A X} / f_{16}$. Finally, from the instability equation (15), the characteristic size of the wave numbers of the dark matter $\phi$ and dark radiation $X_{\mu}$ can be estimated as

$$
k_{*} \sim g_{X X} \dot{\theta} \sim g_{X X}\left(\frac{a\left(\tau_{\mathrm{osc}}\right)}{a\left(\tau_{X}\right)}\right)^{1 / 2} a\left(\tau_{\mathrm{osc}}\right) m_{\phi} .
$$

Following Ref. [46], we performed lattice calculations to examine the evolution of $r(\tau), \epsilon(\tau)$, and $b(\tau)$ for the benchmark point with $g_{X X}=100$ [48]. When performing the lattice simulation, we ignore the evolution due to magnetohydrodynamics, i.e., $\boldsymbol{v}=0$, which we will discuss shortly. Taking various different values of $m_{\phi}$ and $f$, we confirmed that $r$ and $\epsilon$ are indeed insensitive to $m_{\phi}$ and $f$, while $b$ depends on $m_{\phi}$ as in Eq. (19). In Fig. 1, we depict the results for $g_{X X}=100$ and $m_{\phi}=10^{-17} \mathrm{eV}$, showing $a\left(\tau_{X}\right) / a\left(\tau_{\text {osc }}\right) \simeq 5$ and the asymptotic values $r \simeq 0.03$, $\epsilon \simeq 4, b \simeq 7 \times 10^{-18}$ at $\tau \gg \tau_{X}$. The power spectrum of the magnetic field $B$ is plotted in Fig. 2, which exhibits a single spectral peak. [The spectrum with larger box size (solid red) exhibits fake enhancement at the cutoff wave number. This is caused by the mode-mode coupling of the dark photon and ALP fluctuations, which transfers energy towards high wave numbers.] From those results, we find the dark and ordinary magnetic fields given in Eqs. (7) and (8) with the correlation length

$$
\lambda=\frac{2 \pi}{k_{*}} \simeq 0.3 \mathrm{kpc} m_{-17}^{-1 / 2} .
$$
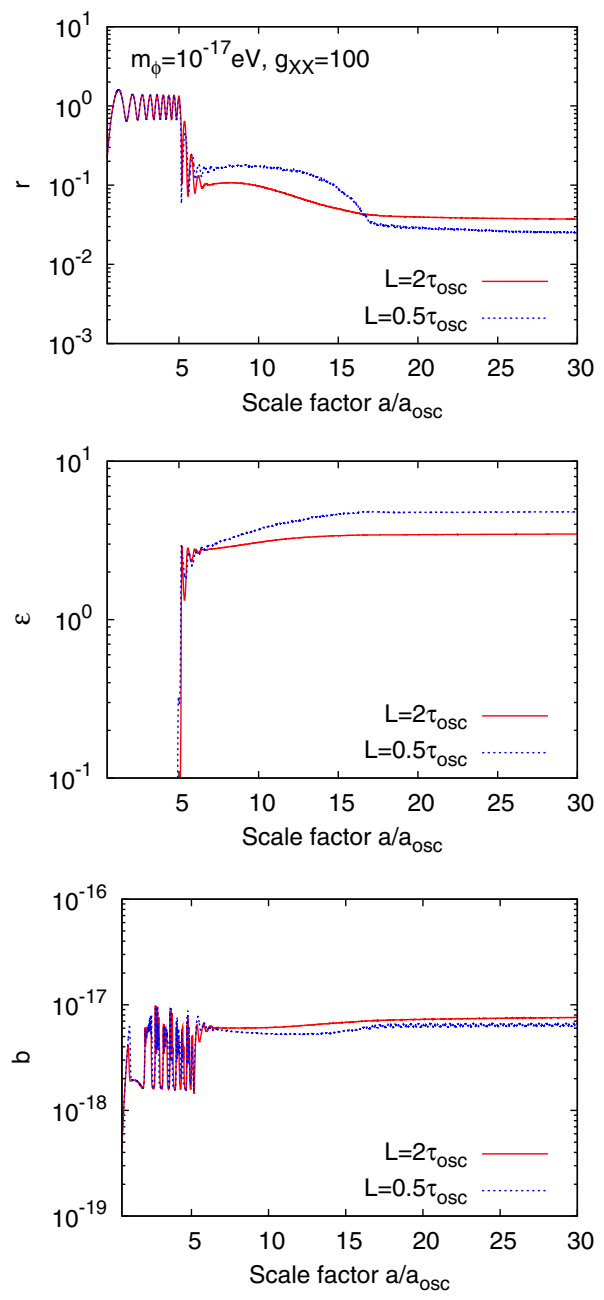

FIG. 1. The time evolution of $r$ (top), $\epsilon$ (middle), and $b$ (bottom) from the lattice calculation with the number of grid $128^{3}$. One can also see $a_{X} / a_{\mathrm{osc}} \simeq 5$ from the epoch when $\epsilon$ and $b$ almost saturate. Note that two different simulation boxes with comoving side lengths $L=2 \tau_{\text {osc }}$ (red solid) and $L=0.5 \tau_{\text {osc }}$ (blue dotted) show consistent results. 


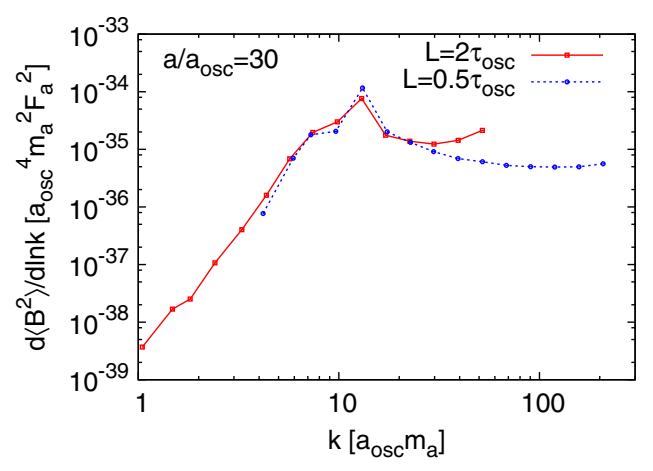

FIG. 2. The power spectrum of produced magnetic fields from the lattice calculation at $a / a_{\text {osc }}=30$. Setup is the same as in Fig. 1. Results from different simulation boxes are consistent around the spectral peak.

The above correlation length is obtained from Fig. 2 and is about 3 times larger than the naive estimation (24). The produced dark and ordinary magnetic fields contribute to the effective number of relativistic degrees of freedom as

$$
\Delta N_{\text {eff }} \simeq 6 \times 10^{-3} m_{-17}^{-1 / 2},
$$

which would be consistent (up to $2 \sigma$ ) with the current observation $N_{\text {eff }}=3.15 \pm 0.23[50]$ for $m_{\phi} \gtrsim 10^{-21} \mathrm{eV}$.

Let us comment on the evolution of $B$ after the production. We expect that the magnetic fields are frozen in after the production. The $B$ fields at kpc scales do not dissipate away even after the recombination due to the high conductivity of the Universe. On the other hand, the Alfvén crossing time, which sets the interaction timescale between magnetic fields and plasma [51], is much larger than the age of the Universe. Thus, it is expected that the $B$ fields are not subject to an evolution due to magnetohydrodynamics.

In our scenario, the dark photon gauge field strengths are inevitably produced on cosmological scales. The produced $B_{X} \sim E_{X}$ can induce a mixing between the ALP, ordinary photon, and dark photon states, which may result in interesting astrophysical or cosmological consequences [31-42,52]. Under $B_{X}$ given in Eq. (7), we find the bound (12) is translated into $g_{A X} \lesssim 5$ when $\phi$ constitutes the dark matter in the Universe. As was noticed in Ref. [40], future measurements of CMB distortions by PIXIE and PRISM can improve the bound on $g_{A X}$ by 2 orders of magnitude. This implies that PIXIE and PRISM will be able to probe the $\mathrm{CMB}$ distortions predicted by $g_{A X}=\mathcal{O}(1)$ which is most favored in our scenario. We note also that for the fuzzy dark matter with $m_{\phi} \sim 10^{-21} \mathrm{eV}$ [53], the resulting value of $\Delta N_{\text {eff }}$ is close to the bound from CMB observation [50], which might be an interesting point in connection with the discrepancy in the values of $H_{0}$ inferred from CMB data and local measurements.

Dark photon fields may contribute to metric perturbations as well. While their characteristic scale $k_{*}$ is beyond the reach of direct cosmological probes (e.g., Lyman- $\alpha$ forests), they can source the acoustic oscillation of photon baryon fluid and contribute to the CMB spectral distortion [54,55], which deserves more detailed study [45].

Our scenario assumes $g_{X X}=\mathcal{O}(10-100)$ and $g_{A X}=\mathcal{O}(1)$. One may ask whether such ALP effective couplings can be obtained from a sensible UV completion of the model. If one assumes that the field range of periodic ALP is of $\mathcal{O}(2 \pi f)$, then naive field theoretic consideration suggests that $g_{X X}=\mathcal{O}\left(e_{X}^{2} / 8 \pi^{2}\right)$ and $g_{A X}=\mathcal{O}\left(e e_{X} / 8 \pi^{2}\right)$, which appear to be significantly smaller than the values assumed in our scenario. This problem can be easily solved by the clockwork mechanism [56-58] enlarging the ALP field range exponentially, while keeping the ALP couplings to gauge fields essentially fixed. An explicit realization along this direction will be presented in the forthcoming paper [45], together with more extensive study of our magnetogenesis scenario.

This work was supported by IBS under the Project No. IBS-R018-D1. T. S. is supported by JSPS KAKENHI Grant Nos. JP15H02082, 18H04339, 18K03640. K. C. thanks K. Kamada, S. Lee, and H. Seong, and T. S. thanks J. Yokoyama, M. Oguri, and T. Shigeyama for fruitful discussions.

*kchoi@ibs.re.kr

†hyungjin.kim@weizmann.ac.il

sekiguti@resceu.s.u-tokyo.ac.jp

[1] P. P. Kronberg, Rep. Prog. Phys. 57, 325 (1994).

[2] D. Grasso and H. R. Rubinstein, Phys. Rep. 348, 163 (2001).

[3] R. Durrer and A. Neronov, Astron. Astrophys. Rev. 21, 62 (2013).

[4] A.-C. Davis, M. Lilley, and O. Tornkvist, Phys. Rev. D 60, 021301 (1999).

[5] A. Brandenburg and K. Subramanian, Phys. Rep. 417, 1 (2005).

[6] A. Neronov and I. Vovk, Science 328, 73 (2010).

[7] F. Tavecchio, G. Ghisellini, L. Foschini, G. Bonnoli, G. Ghirlanda, and P. Coppi, Mon. Not. R. Astron. Soc. 406, L70 (2010).

[8] K. Dolag, M. Kachelriess, S. Ostapchenko, and R. Tomas, Astrophys. J. 727, L4 (2011).

[9] W. Essey, S. Ando, and A. Kusenko, Astropart. Phys. 35, 135 (2011).

[10] A. M. Taylor, I. Vovk, and A. Neronov, Astron. Astrophys. 529, A144 (2011).

[11] K. Takahashi, M. Mori, K. Ichiki, S. Inoue, and H. Takami, Astrophys. J. 771, L42 (2013).

[12] J. D. Finke, L. C. Reyes, M. Georganopoulos, K. Reynolds, M. Ajello, S. J. Fegan, and K. McCann, Astrophys. J. 814, 20 (2015).

[13] M. S. Turner and L. M. Widrow, Phys. Rev. D 37, 2743 (1988).

[14] B. Ratra, Astrophys. J. 391, L1 (1992). 
[15] V. Demozzi, V. Mukhanov, and H. Rubinstein, J. Cosmol. Astropart. Phys. 08 (2009) 025.

[16] N. Barnaby, R. Namba, and M. Peloso, Phys. Rev. D 85, 123523 (2012).

[17] T. Fujita and S. Mukohyama, J. Cosmol. Astropart. Phys. 10 (2012) 034.

[18] T. Fujita and R. Namba, Phys. Rev. D 94, 043523 (2016).

[19] P. Adshead, J. T. Giblin, T. R. Scully, and E. I. Sfakianakis, J. Cosmol. Astropart. Phys. 10 (2016) 039.

[20] T. Vachaspati, Phys. Lett. B 265, 258 (1991).

[21] K. Enqvist and P. Olesen, Phys. Lett. B 319, 178 (1993).

[22] F. Miniati, G. Gregori, B. Reville, and S. Sarkar, Phys. Rev. Lett. 121, 021301 (2018).

[23] K. Kaneta, H.-S. Lee, and S. Yun, Phys. Rev. Lett. 118, 101802 (2017).

[24] V. Iršič, M. Viel, M. G. Haehnelt, J. S. Bolton, and G. D. Becker, Phys. Rev. Lett. 119, 031302 (2017).

[25] K. Takahashi, K. Ichiki, and N. Sugiyama, Phys. Rev. D 77, 124028 (2008).

[26] M. Giovannini and N. Q. Lan, Phys. Rev. D 80, 027302 (2009).

[27] L. Hollenstein, R. K. Jain, and F. R. Urban, J. Cosmol. Astropart. Phys. 01 (2013) 013.

[28] Our model obviously satisfies the recent experimental bounds on ALP couplings from the nuclear spin process [29], as in our case, the quantized ALP-gluon coupling is exactly zero, and the derivative couplings of ALP to nucleons, which are radiatively induced by $g_{A A}$, are much smaller than the bound of Ref. [29].

[29] C. Abel et al., Phys. Rev. X 7, 041034 (2017).

[30] G. Raffelt and L. Stodolsky, Phys. Rev. D 37, 1237 (1988).

[31] D. Wouters and P. Brun, Astrophys. J. 772, 44 (2013).

[32] M. Berg, J. P. Conlon, F. Day, N. Jennings, S. Krippendorf, A. J. Powell, and M. Rummel, Astrophys. J. 847, 101 (2017).

[33] J. P. Conlon, F. Day, N. Jennings, S. Krippendorf, and M. Rummel, J. Cosmol. Astropart. Phys. 07 (2017) 005.

[34] L. Chen and J. P. Conlon, arXiv:1712.08313.

[35] M. C. D. Marsh, H. R. Russell, A.C. Fabian, B. P. McNamara, P. Nulsen, and C. S. Reynolds, J. Cosmol. Astropart. Phys. 12 (2017) 036.

[36] L. Ostman and E. Mortsell, J. Cosmol. Astropart. Phys. 02 (2005) 005.

[37] A. Avgoustidis, C. Burrage, J. Redondo, L. Verde, and R. Jimenez, J. Cosmol. Astropart. Phys. 10 (2010) 024.

[38] P. Tiwari, Phys. Rev. D 95, 023005 (2017).
[39] A. Mirizzi, G. G. Raffelt, and P. D. Serpico, Phys. Rev. D 72, 023501 (2005).

[40] H. Tashiro, J. Silk, and D. J. E. Marsh, Phys. Rev. D 88, 125024 (2013).

[41] A. Mirizzi, J. Redondo, and G. Sigl, J. Cosmol. Astropart. Phys. 08 (2009) 001.

[42] S. Mukherjee, R. Khatri, and B. D. Wandelt, J. Cosmol. Astropart. Phys. 04 (2018) 045.

[43] Note that the bound of Ref. [44] based on WMAP observation does not apply to our case as Ref. [44] assumes that $\boldsymbol{B}$ fields have a power-law spectrum and coherent length $\sim \mathrm{Mpc}$, which are not shared by the dark photon field strength in our scenario.

[44] P. Tiwari, Phys. Rev. D 86, 115025 (2012).

[45] K. Choi, K. Kamada, H. Kim, S. Lee, H. Seong, and T. Sekiguchi (to be published).

[46] N. Kitajima, T. Sekiguchi, and F. Takahashi, Phys. Lett. B 781, 684 (2018).

[47] For $g_{X X} \gtrsim \mathcal{O}(10)$, the initial $\rho_{\phi}$ is abruptly converted to $\rho_{X}$ at $\tau \sim \tau_{X}$, which is determined mostly by $g_{X X}$. After this abrupt conversion, $\left\langle\rho_{\phi}\right\rangle$ and $a(\tau)\left\langle\rho_{X}\right\rangle$ evolve like $\left(\rho_{\phi}\right)_{g=0} \propto 1 / a^{3}(\tau)$. As a consequence, their ratios, i.e., $r$ and $\epsilon$, are insensitive to $m_{\phi}$ and $f$, while there can be a logarithmic dependence which will be ignored here.

[48] See Supplemental Material at http://link.aps.org/ supplemental/10.1103/PhysRevLett.121.031102 for the details of lattice simulation, which includes Refs. [44,46].

[49] K. Yee, IEEE Trans. Antennas Propag. 14, 302 (1966).

[50] P. A. R. Ade et al. (Planck Collaboration), Astron. Astrophys. 594, A13 (2016).

[51] R. Banerjee and K. Jedamzik, Phys. Rev. D 70, 123003 (2004).

[52] D. Ejlli, Eur. Phys. J. C 78, 63 (2018).

[53] W. Hu, R. Barkana, and A. Gruzinov, Phys. Rev. Lett. 85, 1158 (2000).

[54] J. Chluba and R. A. Sunyaev, Mon. Not. R. Astron. Soc. 419, 1294 (2012).

[55] R. Khatri and R. A. Sunyaev, J. Cosmol. Astropart. Phys. 09 (2012) 016.

[56] K. Choi, H. Kim, and S. Yun, Phys. Rev. D 90, 023545 (2014).

[57] K. Choi and S. H. Im, J. High Energy Phys. 01 (2016) 149.

[58] D. E. Kaplan and R. Rattazzi, Phys. Rev. D 93, 085007 (2016). 